\title{
Effective Purification of Germanium Obtained from Mineral Raw Materials
}

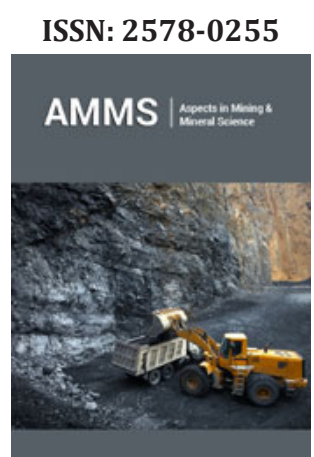

*Corresponding author: Pekar GS, Institute of Semiconductor Physics, National Academy of Sciences, Kiev, Ukraine

Submission: 望 January 20, 2020

Published: 溜February 06, 2020

Volume 4 - Issue 3

How to cite this article: Pekar GS, Singaevsky AF, Lokshyn MM. Effective Purification of Germanium Obtained from Mineral Raw Materials. Aspects Min Miner Sci.4(3). AMMS.000588.2020.

DOI: 10.31031/AMMS.2020.04.000588

Copyright@ Pekar GS, This article is distributed under the terms of the Creative Commons Attribution 4.0 International License, which permits unrestricted use and redistribution provided that the original author and source are credited.

\author{
Pekar GS*, Singaevsky AF and Lokshyn MM \\ Institute of Semiconductor Physics, Ukraine
}

\begin{abstract}
By studying the heat treatment of metallic germanium obtained from mineral raw materials, in is experimentally established that germanium heating in a certain narrow temperature range leads to a significant decrease in the concentration of uncontrolled impurities. The use of such a heating as a preliminary germanium purification makes it possible to increase significantly the efficiency of subsequent germanium purification by a zone melting method.
\end{abstract}

Keywords: Germanium; Purification; Volatile impurities; Zone melting

\section{Introduction}

Single-crystalline and poly-crystalline germanium is one of the main materials of semiconductor technique and is used for fabricating the elements of infrared optics (such as lenses, protective screens, etc.), detectors of ionizing radiation and other devices. Germanium is contained in the earth's crust in the form of a scattered element and may be found in sulfide and iron ores, some coals, almost in all silicates and in a number of minerals (such as argyrodite, confildite, etc.). By means of the use of certain complex and time-consuming operations, germanium may be extracted from the listed materials in the form of oxide $\mathrm{GeO}_{2}$, which may be then chemically reduced with hydrogen at high temperature. Since for most practical applications the high-purity germanium is used as a source material (which subsequently may be doped with certain impurities), processes of metallic germanium purification are very important. Very often the depth of such purification should be very high. For example, to provide germanium transparency in the infrared optical region, i.e. to prepare so-called optical germanium, it should be doped with a certain donor impurity ( $\mathrm{Sb}$, $\mathrm{Na}$, etc.) at a concentration from $5 \cdot 10^{13}$ to $3 \cdot 10^{14} \mathrm{~cm}^{-3}[1,2]$. Therefore, the concentration of uncontrolled impurities in the source germanium before its doping should not exceed at least $10^{12} \mathrm{~cm}^{-3}$. As to detector germanium, it should even be much better refined.

As a main method for germanium purification, zone melting is used. It makes it possible to prepare germanium, which is one of the most chemically pure materials known. However, during zone melting, each germanium ingot must be subjected to repeated purification, i.e. the container with germanium should be many times pulled through a narrow zone of melted germanium. This process is rather long, laborious and energy consuming. Obviously that to increase the efficiency of zone melting, it is desirable to use as much as possible purified source material. In this paper we describe the effect observed by us [3] that makes it easy to lower the content of impurities in the source germanium prior to its purification by zone melting. During a long time we successfully used this effect when preparing the initial material for growing large optical germanium crystals used in modern thermal imaging devices [4].

\section{Method of Preliminary Purification}

Germanium powder obtained by reducing germanium oxide in a hydrogen flow at a temperature about $650-675^{\circ} \mathrm{C}$, weighing approximately $2 \mathrm{~kg}$, was placed in a graphite crucible and heated in a closed chamber under a pressure of $10^{-3} \mathrm{~atm}$ with continuous pumping. After the powder was completely melted, the melt was heated to a temperature of $1050-1150{ }^{\circ} \mathrm{C}$. It was found that in this temperature range a quite narrow region existed, up to $20^{\circ} \mathrm{C}$ wide, in which a rather unexpected process was observed that looked like boiling of the melt. This 
process lasted for about 5-10min. Temperature, corresponding to this region, was different depending on the nature of purified germanium. We explained this process by decomposition and evaporation of some chemical compounds whose composition we could not determine. It seems naturally to suggest that such compounds were initially contained in the germanium dioxide, from which the metal germanium was obtained. As a result of the described process, the content of electrically active impurities in the obtained crystalline germanium dropped by about 5-10 times compared with the material in which the heating in the described narrow temperature region was not carried out. Note that the concentration of electrically active impurities in the obtained germanium ingots was evaluated, as is often done, from the measured value of the electrical resistivity.

It was found that after the subsequent zone melting of the preliminary purified germanium (at 6 passes of the melted zone) the resistivity of almost the entire germanium ingot was approximately $47 \mathrm{Ohm} \cdot \mathrm{cm}$ at room temperature, which corresponded to the intrinsic electrical resistivity of germanium [5] and was indicative of its high purity. At the same time, zone purification of germanium, which was not subjected to the preliminary purification described above, resulted in obtaining ingots with a resistivity about 47 $\mathrm{Ohm} \cdot \mathrm{cm}$ only in the front half of each ingot even after 12-fold passage of the melted zone. As for the rest portions of the ingots, their resistivity did not achieve this value even after the multifold zone-melting purification.

\section{Conclusion}

It is shown that heating of the melted metallic germanium obtained from mineral raw materials, carried out in the experimentally determined narrow (approximately $20^{\circ} \mathrm{C}$ wide) temperature region, results in a significant decrease of the concentration of contaminants. This results in a significant increase in the efficiency of the subsequent purification of this material by zone melting. The described technique has been successfully used for preparing germanium ingots used as a source material for growing, on an industrial scale, of optical germanium crystals for thermal imaging systems.

\section{References}

1. Pekar GS, Singaevsky AF (2012) Na-doped optical germanium bulk crystals. Applied Physics A 108(3): 657-664.

2. Pekar GS, Singaevsky AF (2017) Solubility, diffusion and electrical activity of $\mathrm{Na}$ in bulk Ge crystals. Materials Science in Semiconductor Processing 64: 10-15.

3. Pekar GS, Singaevsky AF, Lokshin MM (2011) Method for obtaining of metal germanium from the germanium dioxide. Patent of Ukraine № 94562.

4. Pekar GS, Singaevsky AF, Lokshin MM, Gordienko VI, Mazurin IV (2018) Large polycrystalline optical germanium Ge: Na plates with improved optical parameters and their application. Semiconductor physics, quantum electronics and optoelectronics 19: 23-27.

5. Smith RA (1978) Semiconductors. ( $2^{\text {nd }}$ edn), Cambridge University Press, UK, p. 540. 